Research Article

\title{
Guardian Map Approach to Feasible Range of Static Stability Margin of Hypersonic Flight Vehicles with Input Saturation
}

\author{
Hao Lei, ${ }^{1}$ Boyi Chen $\left(\mathbb{D},{ }^{1,2,3}\right.$ Yanbin Liu $\mathbb{D}^{1,2,3}$ and Yuping Lu ${ }^{1}$ \\ ${ }^{1}$ College of Automation Engineering, Nanjing University of Aeronautics and Astronautics, Nanjing 210016, China \\ ${ }^{2}$ Academy of Astronautics, Nanjing University of Aeronautics and Astronautics, Nanjing 210016, China \\ ${ }^{3}$ Laboratory of Aerospace Entry, Descent and Landing Technology, Nanjing University of Aeronautics and Astronautics, \\ Nanjing 210016, China
}

Correspondence should be addressed to Boyi Chen; cby_1989@126.com

Received 30 May 2020; Revised 28 June 2020; Accepted 9 July 2020; Published 1 August 2020

Academic Editor: Xiangwei Bu

Copyright $(2020$ Hao Lei et al. This is an open access article distributed under the Creative Commons Attribution License, which permits unrestricted use, distribution, and reproduction in any medium, provided the original work is properly cited.

\begin{abstract}
Static stability margin is a critical parameter in flight control design. The feasible range of it must cover the uncertainty through the flight. To reasonably identify the feasible range of static stability margin in advance, an approach based on guardian maps is proposed for flight control of hypersonic flight vehicles with input saturation. First, the model of hypersonic flight vehicle (HFV) is established as a parametric plant. Then, flying quality requirements for the closed-loop system are formulated as inequality constraints using guardian maps. Moreover, by using linear matrix inequality, the saturation of elevators is taken into account in the integrated control of attitude control. The prescribed minimum of static stability margin that ensures the flying quality of hypersonic flight vehicles with input saturation is obtained. Furthermore, from the prospective of integrated control, it is shown that the feasible range of static stability margin can be enlarged by changing aerodynamic characteristics. The effectiveness of the proposed approach is validated by numerical simulation.
\end{abstract}

\section{Introduction}

Large flight envelopes [1], unstable longitudinal dynamics with input saturation [2], and environmental uncertainty [3] lead to a great of difficulty in flight control system design of hypersonic flight vehicles (HFV). Besides, tracking control for HFV with model uncertainties for prescribed performance is a challenging task [4]. Plenty of advanced control methods have been investigated to address the flight control problems $[5,6]$. The majority focuses on the adaptive nonlinear approach for non-affine models with mismatched disturbance $[7,8]$ and large-envelope tracking problem with global stability and prescribed performance [9-11]. The global stability can be hardly guaranteed in closed-loop system with input saturation since the longitudinal dynamics is inherently unstable for hypersonic flight [12]. Hence, the stability region of a closed-loop system should be confirmed beforehand, and the feasible range in which the closed-loop performance remains must be identified [13].
Static stability margin is a critical parameter in overall design and dramatically affects the performance of flight control system. It may be actively determined by the designer or passively changed according to the flying environment. Releasing static stability margin can improve the flight performance. However, relaxed static stability margin results in stability problem in the flight control system, especially for an unstable plant with limited control authority [14]. The achievable flight stability and performance is closely related to the control authority [15], especially input saturation, control rate, and control failure [16]. Many anti-windup strategies have been proposed, such as introducing auxiliary dynamic systems to regulate the inputs [17]. Instability with input constraints of HFV inflicts serious challenge on the flight control system design. Besides, both the aerodynamic centre and the centre of gravity vary uncertainly with the change of flight environment. Hence, it will be extremely useful for aircraft design and control that the feasible range of static stability margin (or minimum static stability margin) is determined in advance. 


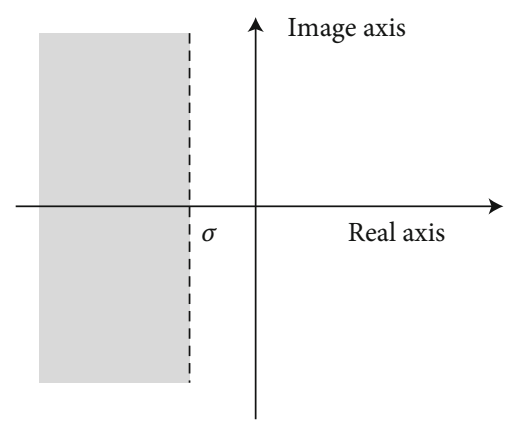

(a) $\sigma$-shifted half-plane

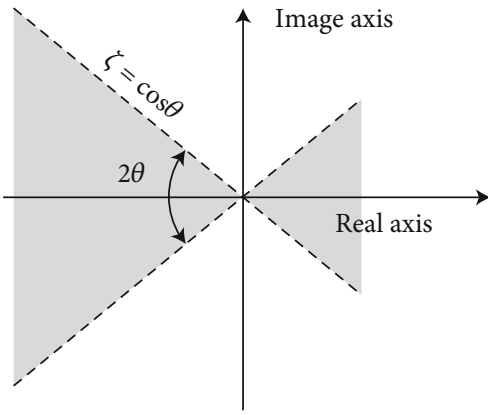

(b) Conic sector with $\theta$

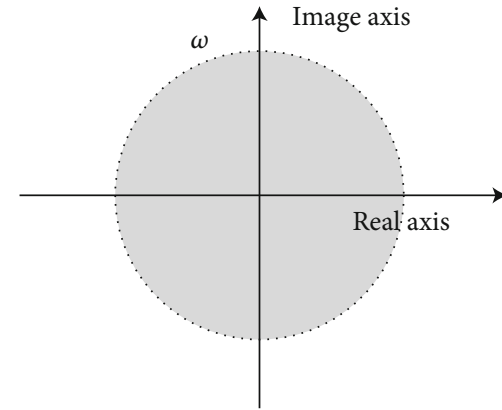

(c) Circle of radius $\omega$

Figure 1: Classical regions $\Omega$ in the complex plane.

Guardian maps were introduced by Saydy et al. as a unifying tool for the study of generalized stability of parameterized families of matrices or polynomials [18]. The guardian map approach for control system design identifies the boundary of controller gain with a suitable initial solution [19]. A feasible solution of the controller gains is restricted by inequalities if the topological structure of the null space for guardian maps remains. According to this property, Sausslé et al. proposed a longitudinal flight control method with handling quality requirements, where the controller gains are automatically tuned using guardian map approach [20]. What is more, guardian maps can be utilized in scheduling or switching algorithms for controller gains over a wide flight range $[21,22]$.

The superiority of the guardian map approach lies in its capability to directly identify the stability region [23]. It will be more concise and efficient than solving a bunch of poleplacement problems, especially for high-dimensional parametric systems. We use a linearized HFV model in a cruising flight condition to examine the proposed method. Input saturation is formulated as linear matrix inequality (LMI) [24]. The boundary of static stability margin is identified by solving a generalized eigenvalue problem of LMI [25], and the controller gain that maximizes the robustness against static stability is obtained simultaneously.

Motivated by the aforementioned observations, which summarizes the challenges the control flight control system design of HFV and the capacity of guardian maps, this paper proposes an approach to calculate the feasible region of static stability margin of HFV based on guardian maps. The predominant contributions of this paper can be summarized as follows: (1) Instead of solving a bunch of pole-placement problems, this paper proposes a more concise and selfcontained approach for solving parametric stability problem. (2) Closed-loop performance is analytically formulated using guardian maps, which enables rough assessment of flight control system before the concrete controller design. (3) With the proposed approach, stability concerns can be integrated in the overall design of HFV for more reliable design against the abovementioned control problem in advance.

The remainder of this paper is structured as follows. The longitudinal dynamic model of an HFV with input saturation constraints is considered in Section 2. The basic principles of guardian map theory are briefly reviewed in Section 3, and flying quality requirements are constructed as inequality con- straints on the basis of guardian maps. The procedure for determining the feasible range of static stability margin for a linearized attitude dynamic model is explored in Section 4. Simulation results are discussed in the next section, and conclusion comes at last.

\section{Guardian Map Approach to Stability Analysis}

2.1. Guardian Maps: Brief Review. Guardian map approach was introduced as a unifying tool for the study of generalized stability of parameterized families of matrices or polynomials [18]. The following discussion focuses on families of matrices but is suitable for polynomials as well. Generalized stability of a matrix is defined as that all eigenvalues of the matrix lie in a prescribed region in the complex plane. The set of matrices that features generalized stability $\Omega$ is expressed as follows:

$$
S_{\Omega}=\left\{\mathbf{A} \in \mathbf{R}^{n \times n} ; \lambda(\mathbf{A}) \subset \Omega\right\},
$$

where $\Omega$ is an open subset of the complex plane, and $\lambda(\mathbf{A})$ denotes the set of eigenvalues of matrix A. A matrix, whose eigenvalues are all located in $\Omega$, is said to be $\Omega$-stable. Hence, $S_{\Omega}$ represents the set of all matrices that are $\Omega$-stable.

Guardian map is a scalar-value map defined in the set $\mathbf{R}^{n \times n}$, which is denoted as $v$. A map $v$ guards $S_{\Omega}$ or $v$ is a guardian map of $S_{\Omega}$, when the following equivalence holds for $\forall \mathbf{A} \in \partial S_{\Omega}$, where $\partial S_{\Omega}$ denotes the boundary of $S_{\Omega}$ :

$$
\nu(\mathbf{A})=0 \Leftrightarrow \mathbf{A} \in \partial S_{\Omega} .
$$

Guardian maps are used to address the generalized stability problem for parametric families of matrices. The value of a guardian map in the domain $\Omega$ is sign-invariant and vanishes on the boundary of $\Omega$. Let $\mathbf{r}=\left[r_{1}, r_{2}, \cdots, r_{k}\right]^{\mathrm{T}} \in \mathbf{R}_{\mathrm{u}}$ be a parameter vector, where $\mathbf{R}_{\mathrm{u}}$ is a path-wise connected subset of $\mathbf{R}^{\mathrm{k}}$, and $\mathbf{A}(\mathbf{r})$ is a matrix which depends continuously on r. Given an open set $\Omega$ and its guardian map $v_{\Omega}$, we can calculate the boundary conditions for $\mathbf{r}$ that $\mathbf{A}(\mathbf{r})$ lies within $S_{\Omega}$ by solving the null space of corresponding guardian maps.

Herein, we introduce the classical regions $\Omega$ in the complex plane (see Figure 1). The analytical expressions of corresponding guardian maps are expressed as follows. 
TABLE 1: Flying quality boundaries [23].

\begin{tabular}{lcc}
\hline$\zeta_{\text {sp }}$ & $\omega_{\text {sp }}$ & $\omega_{\text {BW } \theta}$ \\
\hline $0.35 \sim 1.30$ & $>0.87 \mathrm{rad} / \mathrm{s}$ & $>1.5 \mathrm{rad} / \mathrm{s}$ \\
\hline
\end{tabular}

$$
\begin{aligned}
& v_{\sigma}(\mathbf{A})=\operatorname{det}(\mathbf{A} \odot \mathbf{I}-\sigma \mathbf{I} \odot \mathbf{I}) \operatorname{det}(\mathbf{A}-\sigma \mathbf{I}), \\
& v_{\zeta}(\mathbf{A})=\operatorname{det}\left[\mathbf{A}^{2} \odot \mathbf{I}+\left(1-2 \zeta^{2}\right) \mathbf{A} \odot \mathbf{A}\right] \operatorname{det}(\mathbf{A}), \\
& v_{\omega}(\mathbf{A})=\operatorname{det}\left(\mathbf{A} \odot \mathbf{A}-\omega^{2} \mathbf{I} \odot \mathbf{I}\right) \operatorname{det}\left(\mathbf{A}^{2}-\omega^{2} \mathbf{I}\right),
\end{aligned}
$$

where $\odot$ denotes the bialternate product [26].

Guardian map of any region that is generated by the intersection of these classic domains can be expressed as the product of the above functions. Considering requirements on flying quality are mainly defined as time constant, damping ratio, and natural frequency, the performance of flight control system can be addressed by guardian maps in terms of generalized stability.

2.2. Generalized Stability Defined by Flying Quality. Practical considerations related to damping ratio, bandwidth, and vehicle handling quality can be expressed as the generalized stability. The flying quality (FQ) criteria for the shortperiod mode considered in this work are the damping ratio $\zeta_{\mathrm{sp}}$, natural frequency $\omega_{\mathrm{sp}}$, and pitch attitude bandwidth $\omega_{\mathrm{BW} \theta}$. Boundaries for these criteria defined by military standards are listed as follows (see Table 1).

The equivalent form of the short-period characteristic polynomial includes the information of $\zeta_{\mathrm{sp}}$ and $\omega_{\mathrm{sp}}$. Let $p_{1}$ and $p_{2}$ be the poles of short-period characteristic polynomial; then, $\zeta_{\mathrm{sp}}$ and $\omega_{\mathrm{sp}}$ can be expressed by the locations of the poles as follows:

$$
\begin{gathered}
\zeta_{\mathrm{sp}}=\frac{p_{1}+p_{2}}{2 \sqrt{p_{1} p_{2}}}, \\
\omega_{\mathrm{sp}}=\sqrt{p_{1} p_{2}} .
\end{gathered}
$$

If the short-period mode contains a pair of complex poles, the damping ratio is inherently less than 1; then, the $\mathrm{FQ}$ requires $\zeta_{\mathrm{sp}}>0.35$. If the short-period mode has two real poles, then the requirement of damping ratio $\zeta_{\mathrm{sp}}<1.3$ implies that $0.22<p_{1} / p_{2}<4.54$. Therefore, the level 1 requirements of FQ are then represented as the generalized stability defined in complex plane (see Figure 2). The corresponding guardian map is formulated as follows:

$$
v_{\Omega}(\mathbf{A})=v_{\sigma}(\mathbf{A}) v_{\zeta}(\mathbf{A}) v_{\omega}(\mathbf{A})
$$

where $\sigma=-0.87, \zeta=0.35$, and $\omega=3.95$.

Guardian maps provide an extremely powerful tool to parametric stability analysis. They extend the Hurwitz's stability to any desired region in complex plane, which affords the robust analysis of closed-loop performance against parametric uncertainty. The formulation of parametric plant is then presented in the next section.

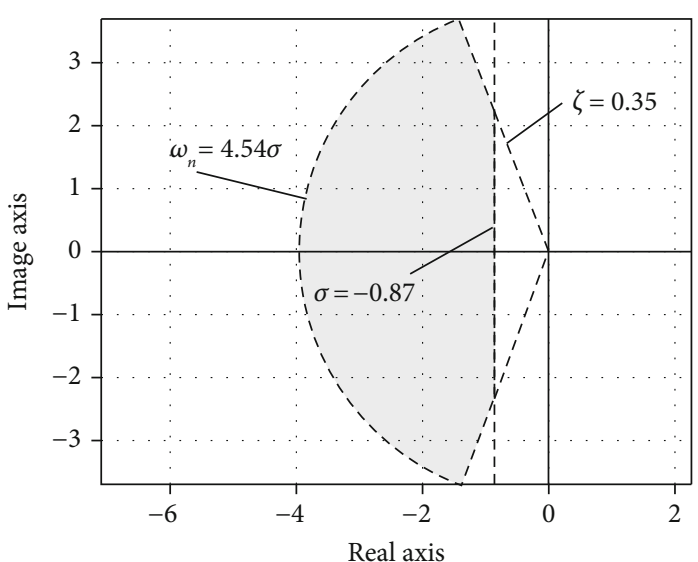

FIgURE 2: General stability target domain satisfying FQ requirements.

\section{Parametric Model with Input Saturation}

In this work, we apply a reduced complexity controloriented model (COM) for control design and stability analysis, and a high-fidelity simulation model (SM) for numerical simulation [27].

3.1. Longitudinal Dynamics with Input Saturation. This longitudinal dynamic model includes five rigid-body state variables, namely, velocity $V$, altitude $h$, angle of attack $\alpha$, pitch rate $q$, and pitch angle $\theta$. Two control inputs, namely, elevator deflection $\delta_{\mathrm{e}}$ and fuel equivalent ratio (FER) $\phi$, are utilized. The equations of motion of longitudinal dynamics derived by Lagrange's equations are written in the stability axes as follows [28]:

$$
\begin{aligned}
& \dot{V}=\frac{T \cos \alpha-D}{m}-g \sin \gamma, \\
& \dot{\alpha}=-\frac{L+T \sin \alpha}{m V}+\frac{g}{V} \cos \gamma+q, \\
& \dot{q}=\frac{M}{I_{y y}}, \\
& \dot{\theta}=q, \\
& \dot{h}=V \sin \gamma,
\end{aligned}
$$

where $m$ and $I_{y y}$ denote the vehicle mass and moment of inertia, respectively. $\gamma=\theta-\alpha$ is the flight path angle (FPA). $L, D, M$, and $T$ represent lift, drag, pitch moment, and thrust, respectively. The expressions of forces and moments that act on the vehicle are as follows:

$$
\begin{aligned}
L & \approx \bar{q} S C_{L}, \\
D & \approx \bar{q} S C_{D}, \\
M & \approx \bar{q} S c C_{m}+z_{T} T, \\
T & \approx \bar{q} C_{T},
\end{aligned}
$$


where $\bar{q}=\rho V^{2} / 2$ denotes the dynamic pressure, $S$ denotes the reference area, $c$ denotes the mean aerodynamic chord, and $z_{T}$ denotes the shift distance, which accounts for the pitching moment produced by the underslung scramjet engine.

The flight control system of HFV focuses on the longitudinal stability of the short period mode, namely, attitude dynamics. Attitude dynamics can be written in the following compact form:

$$
\dot{\mathbf{x}}=\mathbf{f}_{\mathrm{a}}\left(\mathbf{x}_{\mathrm{a}}, \mathbf{x}_{\mathrm{l}}, \mathbf{p}\right)+\mathbf{g}_{\mathrm{a}}\left(\mathbf{x}_{\mathrm{l}}, \mathbf{p}\right) \delta_{\mathrm{e}},
$$

where $\mathbf{x}_{\mathrm{a}}=[\alpha, q]^{\mathrm{T}}$ is the state of the short-period mode, $\mathbf{x}_{1}=$ $[V, h, \gamma]^{\mathrm{T}}$ is the state of the phugoid mode, and $\mathbf{p}$ is the parameter vector that includes other variables. The linearized state space representation of Equation (15) is

$$
\left[\begin{array}{c}
\Delta \dot{\alpha} \\
\Delta \dot{q}
\end{array}\right]=\left[\begin{array}{cc}
-\frac{L_{\alpha}+T_{\alpha} \sin \alpha}{V}-\frac{T \cos \alpha}{m V} & 1 \\
M_{\alpha}+z_{T} T_{\alpha} & 0
\end{array}\right]\left[\begin{array}{c}
\Delta \alpha \\
\Delta q
\end{array}\right]+\left[\begin{array}{c}
-\frac{L_{\delta}}{V} \\
M_{\delta}
\end{array}\right] \Delta \delta_{\mathrm{e}}
$$

where the dimensional derivatives are calculated from the nondimensional derivatives according to

$$
\begin{aligned}
L_{\alpha} & =\frac{\bar{q} S}{m} C_{L \alpha}, \\
L_{\delta} & =\frac{\bar{q} S}{m} C_{L \delta}, \\
M_{\alpha} & =\frac{\bar{q} S c}{I_{y y}} C_{m \alpha}, \\
M_{\delta} & =\frac{\bar{q} S c}{I_{y y}} C_{m \delta}, \\
T_{\alpha} & =\frac{\bar{q}}{m} C_{T \alpha} .
\end{aligned}
$$

One of the most detrimental factors for the control system design of HFVs is input amplitude saturation. The nonlinearity of actuator dynamics leads to a stability problem, especially for unstable plants. The elevator deflection saturation considered in this work is formulated as follows:

$$
\delta_{\mathrm{e}}=\operatorname{sat}\left[\delta_{\mathrm{r}}(t)\right]= \begin{cases}\delta_{\max }, & \delta_{\mathrm{r}}(t) \geq \delta_{\max } \\ \delta_{\mathrm{r}}(t), & \delta_{\max }>\delta_{\mathrm{r}}(t)>\delta_{\min } \\ \delta_{\min }, & \delta_{\mathrm{r}}(t) \leq \delta_{\min }\end{cases}
$$

The elevator deflection for straight and level flight is denoted as $\delta_{s}$. The control input of attitude dynamics for a level flight is $\Delta \delta_{\mathrm{e}}(t)=\delta_{\mathrm{e}}(t)-\delta_{\mathrm{s}}$. Thus, the input amplitude saturation is the gap between the steady elevator deflection and the maximum or minimum deflection of the elevator. We assume that the input amplitude saturation possesses symmetrical characteristic, that is

$$
\Delta \bar{\delta}_{\mathrm{e}}=\Delta \delta_{\max }=-\Delta \delta_{\min }=\min \left\{\left|\delta_{\max }-\delta_{s}\right|,\left|\delta_{s}-\delta_{\min }\right|\right\} .
$$

The saturation of elevator deflection Equation (11) is formulated as inequality constraints as follows:

$$
\Delta \delta_{\mathrm{e}}^{2}(t) \leq \Delta \bar{\delta}_{\mathrm{e}}^{2}, \quad \forall t \in[0, \infty) .
$$

3.2. Parametric Model for Static Stability Margin. The longitudinal stability is significantly influenced by the distance between the centre of gravity (CG) and the centre of pressure (CP). Static stability margin is defined as $K_{n}=\left(x_{\mathrm{CP}}-x_{\mathrm{CG}}\right) / c$ to qualify stability, where $x_{\mathrm{CP}}$ is the axial distance form $\mathrm{CP}$ to the leading edge of the fuselage, and $x_{C G}$ is the axial distance from the CG. A negative static stability margin indicates an instable plant.

According to the flight dynamics of aircraft, the nondimensional stability derivative $C_{m \alpha}$ and control derivative $C_{m \delta}$ can be expressed through $K_{n}$ :

$$
\begin{aligned}
C_{m \alpha} & =\frac{\partial C_{m}}{\partial C_{L}} \frac{\partial C_{L}}{\partial \alpha}=-K_{n} C_{L \alpha}, \\
C_{m \delta} & =\frac{\partial C_{m}}{\partial C_{L}} \frac{\partial C_{L}}{\partial \delta}+\frac{\partial C_{m}}{\partial \delta}=-K_{n} C_{L \delta}+C_{m \delta}^{n},
\end{aligned}
$$

where $C_{m \delta}^{n}$ is the net control derivatives. It excludes the pitching moment produced by the additional lift from elevator deflection.

The objective of this work is to determine the static stability margin boundary with input saturation while maintain the closed-loop performance. The linearized state-space representation of the short-period dynamics (Equation (9)) is parameterized by static stability margin $K_{n}$ and denoted as follows:

$$
\Delta \dot{\mathbf{x}}=\mathbf{A}\left(K_{n}\right) \Delta \mathbf{x}+\mathbf{b}\left(K_{n}\right) \Delta \delta_{\mathrm{e}} .
$$

Closed-loop stability and performance are affected by the feedback gain and $K_{n}$. The feasible range for these parameters maintaining closed-loop performance is obtained by guardian map theory.

The closed-loop system is regarded as a family of matrices with parameterized controller gain $\mathbf{K}$. For the parametric plant as Equation (15), the closed-loop system can be represented as $\mathbf{A}_{c}\left(K_{n}, \mathbf{K}\right)$. FQ that relates to damping ratio and bandwidth can be expressed as generalized stability domain, as shown in Figure 2. The corresponding guardian map is formulated as follows:

$$
v_{\Omega}\left[A_{c}\left(K_{n}, \mathbf{K}\right)\right]=v_{\sigma}\left[A_{c}\left(K_{n}, \mathbf{K}\right)\right] v_{\zeta}\left[A_{c}\left(K_{n}, \mathbf{K}\right)\right] v_{\omega}\left[A_{c}\left(K_{n}, \mathbf{K}\right)\right]
$$

The guardian map approach for control system design identifies the boundary of the controller gain with a suitable initial solution by calculating the roots of Equation (16). Likewise, a feasible solution of the controller gain can be formulated as inequality constraints if the topological structure 


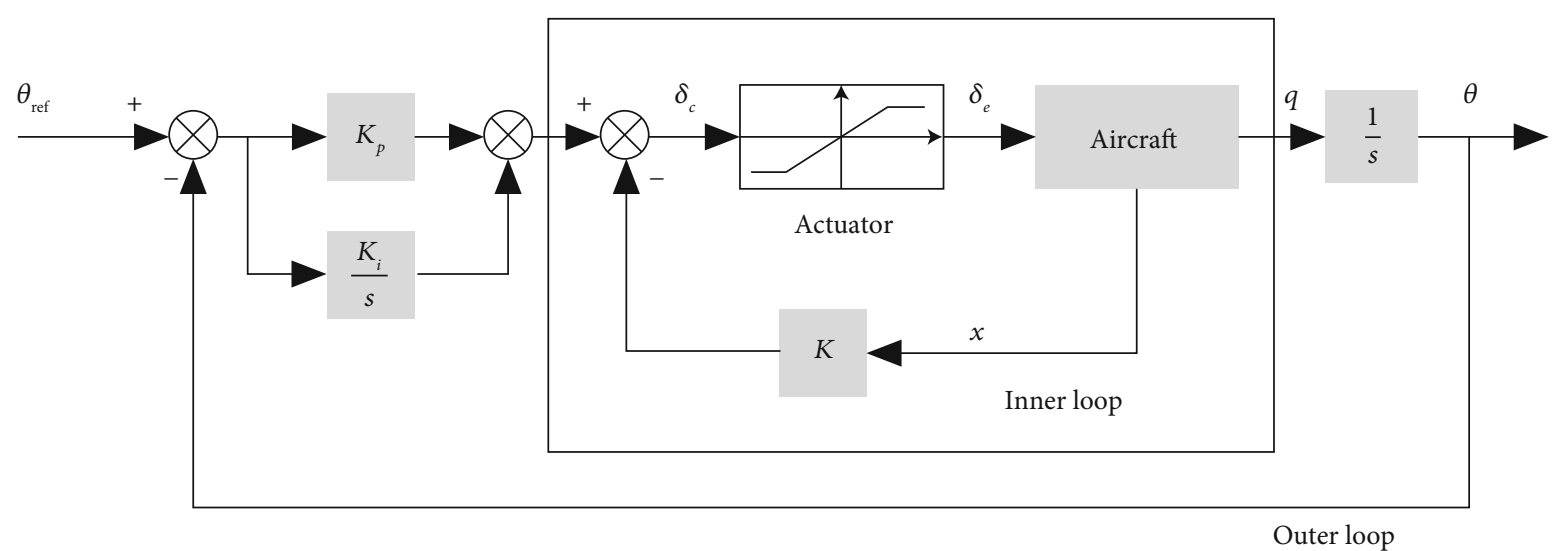

FIgURE 3: Inner-outer loop control system.

of the null space of the guardian maps does not change. Therefore, the determination of the static stability margin boundary of the plant can be solved as a constrained optimization problem:

$$
\begin{array}{cc}
\text { obj. } & \min _{\mathbf{K}}\left\{K_{n}\right\}, \\
& 0>(-1)^{l_{1}} v_{\sigma}\left[A_{c}\left(K_{n}, \mathbf{K}\right)\right], \\
\text { s.t. } & 0>(-1)^{l_{2}} v_{\zeta}\left[A_{c}\left(K_{n}, \mathbf{K}\right)\right], \\
& 0>(-1)^{l_{3}} v_{\omega}\left[A_{c}\left(K_{n}, \mathbf{K}\right)\right],
\end{array}
$$

where $l_{i}, i=1,2,3$ are the controlling parameters for inequality polarity, whose value is set to be 1 or 2 . They are determined by the initial solution and regarded as constants in the optimization. The solution of the constrained optimization problem Equation (17) can be obtained with a numerical optimization algorithm.

However, an elegant solution is acquired based on the generalized eigenvalue problem of LMI when the inequality constraints regarding parameter $K_{n}$ have the linear from of first order. An illustrative example of control system design is provided in the next section.

\section{Static Stability Margin Analysis in the Closed- Loop System}

The following section presents a control methodology that can minimize the static stability margin of longitudinal dynamics. We use a classic decentralized inner-outer loop control system architecture for attitude regulation to illustrate controller design process (see Figure 3). The inner loop controller is full state feedback control, and the outer loop controller is a PI controller. The gains of the inner-loop controller is are donated as $\mathbf{K}=\left[K_{\alpha}, K_{q}\right]$.

4.1. Constraints with Requirements on FQ and Input Saturation. The guardian map for FQ requirements is formulated as multiple products of guardian maps (Equation (16)). The design of controller gain with stability margin $\sigma$ can be formulated as LMI, whereas the other guardian maps with damping ratio and natural frequency are used as nonlinear inequalities. However, these convex inequalities can be converted to an LMI form via Schur complements.

$$
\begin{aligned}
& {\left[\begin{array}{ccc}
-\mathbf{K} \mathbf{c}_{\sigma, 1}+d_{\sigma, 1} & 0 \\
* & -\mathbf{K} \mathbf{c}_{\sigma, 2}+d_{\sigma, 2}
\end{array}\right]>0,} \\
& {\left[\begin{array}{ccc}
-\mathbf{K} \mathbf{c}_{\zeta, 1}+d_{\zeta, 1} & \mathbf{K} & 0 \\
* & \mathbf{a}_{\zeta}^{-1} & 0 \\
* & * & -\mathbf{K} \mathbf{c}_{\zeta, 2}+d_{\zeta, 2}
\end{array}\right]>0,} \\
& {\left[\begin{array}{ccc}
-\mathbf{K}_{\omega, 1}+d_{\omega, 1} & \mathbf{K} & 0 \\
* & \mathbf{a}_{\omega}^{-1} & 0 \\
* & * & -\mathbf{K c}_{\omega, 2}+d_{\omega, 2}
\end{array}\right]>0,}
\end{aligned}
$$

where coefficients $\mathbf{a}_{*}, \mathbf{c}_{*, i}, d_{*, i}, *=\sigma, \zeta, \omega$, and $i=1,2$ are calculated based on the plant dynamics and control specifications $\zeta_{\mathrm{sp}}, \omega_{\mathrm{sp}}$, which are listed in the Appendix.

The input saturation constraint (Equation (13)) can also be formulated as an LMI problem. We assume that the control gain can be factorized as $\mathbf{K}=\mathbf{Y} \mathbf{Q}^{-1}$, and $\mathbf{Q}$ is a definite symmetric matrix.

$$
\begin{aligned}
& {\left[\begin{array}{cc}
\mathbf{Q} & \mathbf{Q} \mathbf{K}^{\mathrm{T}} \\
* & \Delta \bar{\delta}_{\mathrm{e}}^{2} \mathbf{I}
\end{array}\right] \geq 0,} \\
& {\left[\begin{array}{cc}
\mathbf{I} & \mathbf{x}_{0}^{\mathrm{T}} \\
* & \mathbf{Q}
\end{array}\right] \geq 0,}
\end{aligned}
$$

where $\mathbf{x}_{0}$ is the initial state, and $\mathbf{Q}>0$ is the weighting matrix. The LMI constraint implies that for any state that satisfies inequality (Equation (21)), the control input with feedback gain $\mathbf{K}=\mathbf{Y Q}^{-1}$ does not exceed the amplitude saturation $\Delta \bar{\delta}_{e}$.

4.2. Boundary of Static Stability Margin. All coefficients in the LMIs (Equations (18)-(20)) are parameterized by static stability margin $K_{n}$. The inequality constraints (Equations (19) 
TABLE 2: Parameters of the HFV model.

\begin{tabular}{|c|c|c|c|c|}
\hline No & Parameters & Symbols & Nominal values & Units \\
\hline 1 & Fuselage length & $L$ & 100 & $\mathrm{ft}$ \\
\hline 2 & Axial distance of the CG from the leading edge & $x_{\mathrm{CG}} / L$. & 55 & $\%$ \\
\hline 3 & Reference area & $S$ & 17 & $\mathrm{ft}^{2}$ \\
\hline 4 & Mean aerodynamic chord (MAC) & $c$ & 17 & $\mathrm{ft}$ \\
\hline 5 & Shift distance of scramjet engine & $z_{T}$ & 8.36 & $\mathrm{ft}$ \\
\hline 6 & Static stability margin & $K_{n}$ & -55.01 & $\%$ \\
\hline 7 & Stability derivatives & $C_{L \alpha}$ & 4.6773 & $\operatorname{rad}^{-1}$ \\
\hline 8 & Control derivatives & $C_{L \delta}$ & 0.7622 & $\operatorname{rad}^{-1}$ \\
\hline 9 & Net control derivatives & $C_{m \delta}^{n}$ & -1.7090 & $\mathrm{rad}^{-1}$ \\
\hline
\end{tabular}

TABLE 3: Trimmed conditions of SM.

\begin{tabular}{lccccccc}
\hline States & $h(\mathrm{ft})$ & $V(\mathrm{ft} / \mathrm{s})$ & $\alpha(\mathrm{deg})$ & $\theta(\mathrm{deg})$ & $q(\mathrm{deg} / \mathrm{s})$ & $\delta(\mathrm{deg})$ & $\phi(\mathrm{a} . \mathrm{u})$. \\
Values & 85000 & 7702 & 1.2046 & 1.2046 & 0 & 12.6480 & 0.2372 \\
\hline
\end{tabular}

and (20)) are nonlinear regarding $K_{n}$. Therefore, only constraint (Equation (18)) can be converted into parameterized linear inequality form.

$$
\mathbf{A}_{\sigma}(\mathbf{K})<K_{n} \mathbf{B}_{\sigma}(\mathbf{K})
$$

The controller gain that minimizes the static margin is obtained by solving the generalized eigenvalue problem of LMI, and the minimum static stability margin is determined simultaneously.

Hence, the objective of the optimization problem is formulated as the minimization of static margin with respect to controller parameters $\mathbf{K}$ :

$$
\min _{\mathbf{K}}\left\{K_{n}\right\}
$$

The optimization problem (Equation (24)) is constrained by the requirements of FQ and input saturation, which are formulated as inequality constraints:

$$
\begin{gathered}
\mathbf{A}_{\sigma}(\mathbf{K})<K_{n} \mathbf{B}_{\sigma}(\mathbf{K}), \\
0<\mathbf{B}_{\sigma}(\mathbf{K}), \\
0 \leq\left[\begin{array}{cc}
\mathbf{Q} & \mathbf{Q} \mathbf{K}^{\mathrm{T}} \\
* & \Delta \bar{\delta}_{\mathrm{e}}^{2} \mathbf{I}
\end{array}\right] .
\end{gathered}
$$

The controller gain obtained from the proposed problem Equation (24) can only guarantee the control specification on stability margin $\sigma$, while other FQ requirements expressed as guardian maps are examined afterwards.

The gains $K_{\alpha}, K_{q}$ for the inner loop are calculated by solving the generalized eigenvalue problem (Equation (24)). $K_{p}$, $K_{i}$ for the outer loop are tuned automatically based on guardian maps to maintain FQ. The simulation results and analysis are provided in the next section.

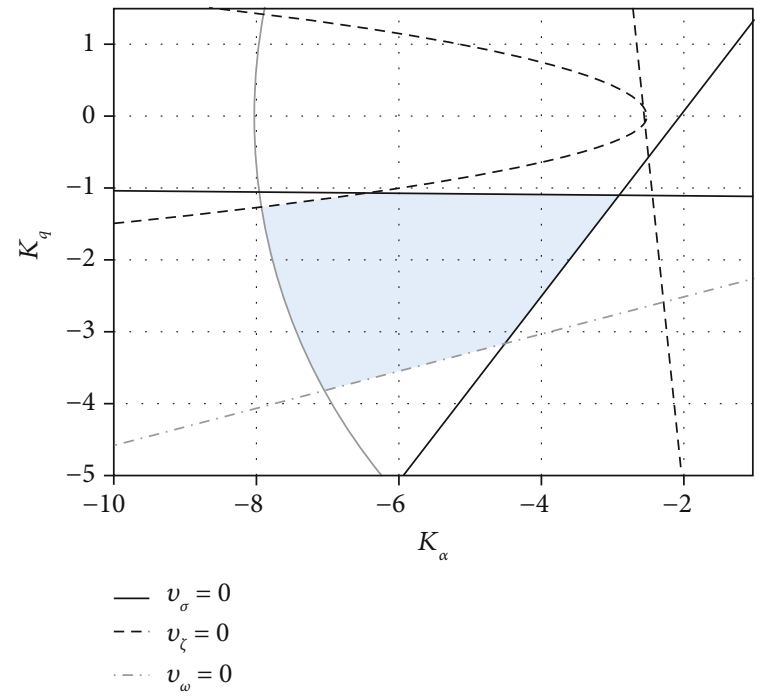

FIGURE 4: Feasible range (gray area) of controller gains satisfying input saturation (solid gray line) and FQ requirements.

\section{Simulation Results}

In this paper, we apply the reduced complexity controloriented model (COM) for robust analysis and control law design, and a high-fidelity simulation model (SM) for numerical simulation. Table 2 provides parameters of the HFV model and estimated values of stability and control derivatives used in this work [27].

Control system design is carried out for the cruising flight condition $h=85000 \mathrm{ft}, M a=7.8497$. The trim conditions are listed in Table 3. The maximum and minimum deflections of the elevators are assumed to be $\pm 20^{\circ}$. Thus, the saturation value of the control input for the cruising flight is $\Delta \bar{\delta}_{e}=$ $7.520^{\circ}$. Compared with pole-placement technique, the superiority of the guardian map approach lies in its capability to 


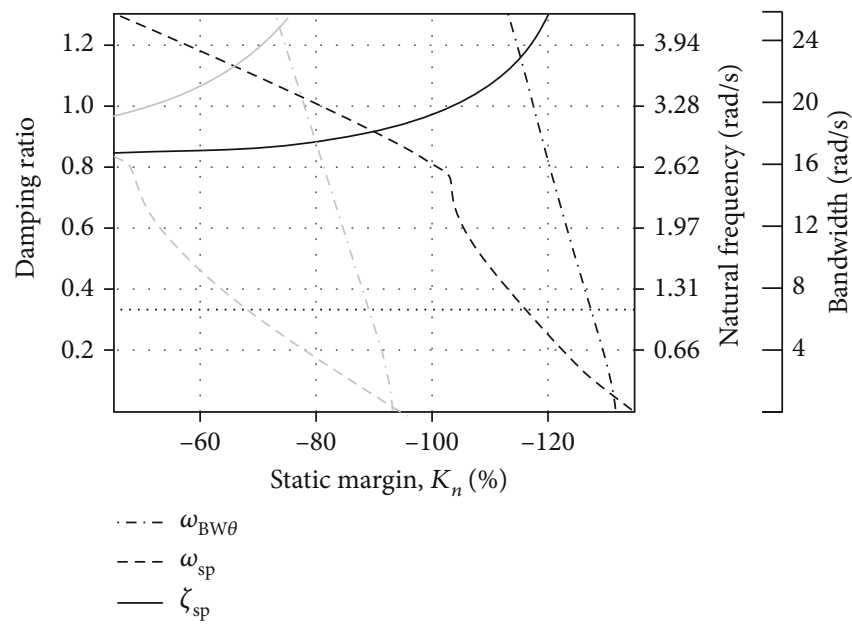

FIGURE 5: FQs of the closed-loop system with different controllers.

automatically define the feasible range of the controller gain instead of a specific value. The feasible range of the controller gain is calculated with LMIs (Equations (18)-(20)) by guardian maps (see Figure 4). The admissible controller gain for a specific plant must lie in the region regardless of the design method, such as LQR methodology or other optimal control design methods.

The feasible range of controller gains varies with the change of static stability margin. The closed-loop system requires a higher feedback gain to stabilize the plant as the decrease in static stability margin. Therefore, the feasible range shrinks accordingly and even vanishes with limited control input. Minimum static stability margin controller (MSMC) design methodology is to find the controller gain that minimizes static stability margin while still maintain FQ of the closed-loop system with limited input. We also provide an illustrative example of the proposed controller for a nonlinear model of the HFV and compare the control performance obtained using MSMC and a linear quadratic regulator (LQR) [29].

5.1. Comparison of $L Q R$ and $M S M C$. The performance of the closed-loop system with two controllers is examined with the decrease in the static stability margin. The lower limit of $\zeta_{\mathrm{sp}}$ and $\omega_{\text {sp }}$ is shown as a dotted line in Figure 5. The closedloop system becomes unstable when the natural frequency is zero. The static stability margin, at which $\omega_{\text {sp }}$ vanish, is regarded as boundary for stable closed-loop system.

A comparison of the feasible range of static stability margin between LQR and MSMC is shown in Figure 6. The dashed-dotted line in Figure 6 indicates the nominal value of the static stability margin of COM. Two feasible ranges of the static stability margin are present. The smaller one defines the boundary that the closed-loop system satisfies FQ requirements, and the larger one establishes the limitation that the close-loop system remains stable with input saturation.

The step responses of the pitch angle of the nonlinear system with different static stability margins are shown in Figure 7. $\zeta_{\mathrm{sp}}>0.35$ is equivalent to that in which the over-

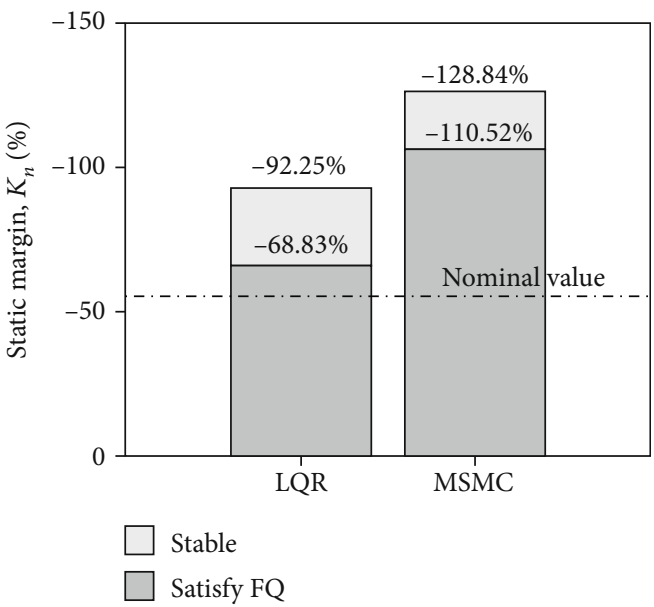

FIgURE 6: Comparison of the admissible static stability margin for specific input saturation.

shoot is less than $28.5 \%$. The performance of the closedloop system with MSMC still satisfies FQ requirements with admissible input, whereas the control input from the LQR is beyond the admissible range, which is shown as dasheddotted lines in Figure 7(b). Furthermore, the overshoot of the step response with LQR control reaches $47 \%$. Although the closed-loop system with LQR does not satisfy FQ, the stability of the closed-loop system can still be maintained with sufficient control authority. Therefore, the primary reason for the instability issue of HFV dynamics is control saturation.

5.2. Feasible Range of Static Stability Margin. Given input saturation, the minimum static stability margin can be determined via the proposed method in the cruising flight condition. The nominal value of $K_{n}$ is $-55 \%$. The minimum static stability margin with input saturation $\Delta \bar{\delta}_{e}=7.520^{\circ}$ in cruising flight condition is $110.52 \%$. Thus, the CG of SM can shift backwards by up to $110.52 \%$. However, the trimmed elevator deflection increases as CG shifts afterwards to counteract the nose-up pitching moment. Therefore, the 

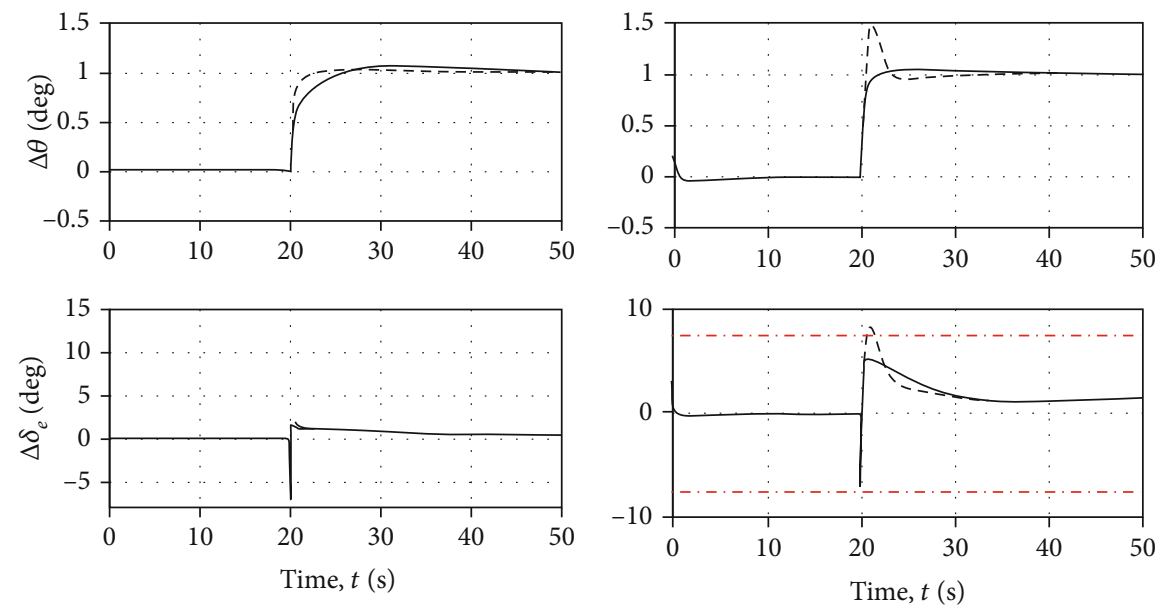

(a) $K_{\mathrm{C}}=-55 \%$

(b) $K_{\mathrm{n}}=-100 \%$

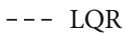

- MSMC

FIgURe 7: Nonlinear simulation of step reference with input saturation.

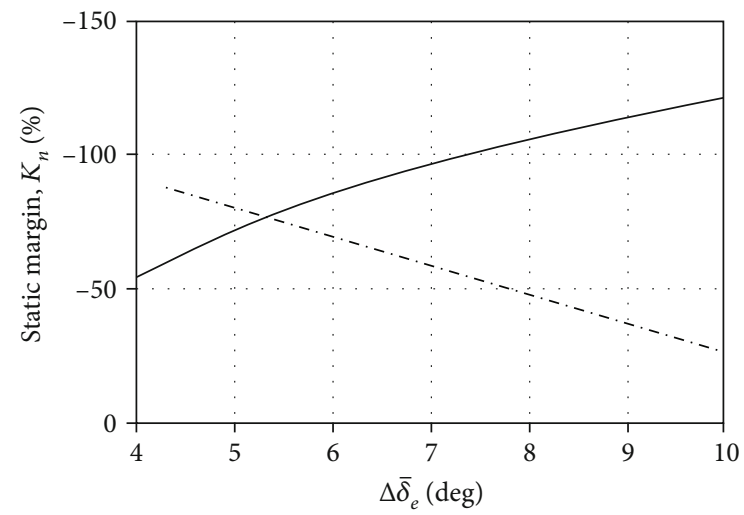

FIGURE 8: Determination of $K_{n}^{\mathrm{min}}$. Solid line: $K_{n}^{\min }$ for specific input saturation. Dashed line: trimmed characteristics.

saturation value of elevator deflection decreases as static stability margin decreases, as shown by the dashed-dotted line in Figure 8.

The interaction point in Figure 8 is regarded as the minimum static stability margin for the cruising flight condition, which is determined to be $-76 \%$. The graphical representation of the HFV model is shown in Figure 9.

The significance of this work is the determination of the feasible range of static stability margin characterized by $K_{n}^{\mathrm{min}}$, which is directly determined by the input saturation and affected by the control derivatives of the model. The effect of the control derivatives on $K_{n}^{\mathrm{min}}$ is investigated in the next subsection.

\section{Impact of Control Derivatives on $K_{n}^{\min }$}

Instability with input constraints of HFV inflicts serious challenge on the flight control system design. The static stability margin is one of the most critical parameters both for overall design and flight control system. With the proposed approach, the lower limit of static stability margin $K_{n}^{\mathrm{min}}$ has been confirmed. However, there may be a mismatch between the prescribed flight performance and stability. Hence, the approach of integrated design emerges to address the issue. This subsection will discuss the influence of aerodynamic characteristics on the feasible range of static stability margin.

The nominal values of the control derivatives of the baseline model are $C_{m \delta}^{0}=1.709 \mathrm{rad}^{-1}$ and $C_{L \delta}^{0}=0.76224 \mathrm{rad}^{-1}$. The variation in the minimum static stability margin over the control derivatives for cruising flight is shown in Figure 10. The most effective approach to improve the control performance by releasing the static stability margin is to adjust the control derivatives along the gradient direction as shown in Figure 10.

The control derivatives are determinant factors affecting the closed-loop stability. The first one is $C_{m \delta}$, followed by the ratio of control derivatives $C_{L \delta} / C_{m \delta}$. The location of the control surfaces is more important than the size in addressing the closed-loop stability. Thus, the control surfaces are expected to be located at the rearward-most position of the vehicle.

\section{Conclusions}

In this paper, an analytical approach for determining the feasible range of static stability margin has been proposed based on guardian maps and linear matrix inequality. The feasible range of static stability margin is characterized by the minimum static stability margin, which is obtained by formulating an optimal problem. Meanwhile, flying quality requirements and input saturation are reconstructed as nonlinear inequalities. Simulation results show the effectiveness of the proposed approach for defining the feasible range of static stability margin. Given the model of hypersonic flight vehicles by Parker, the maximum relative 


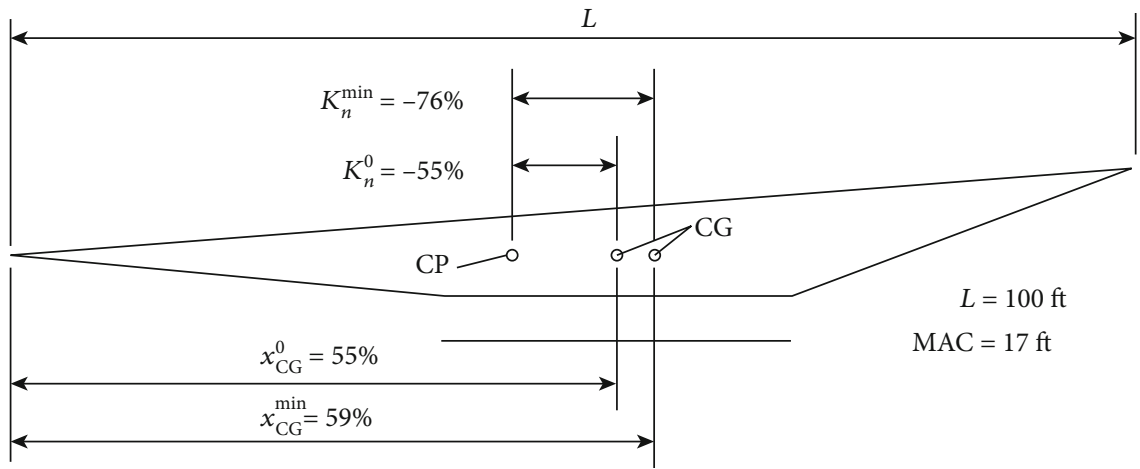

FIGURE 9: Sketch of HFV with the minimum static stability margin.

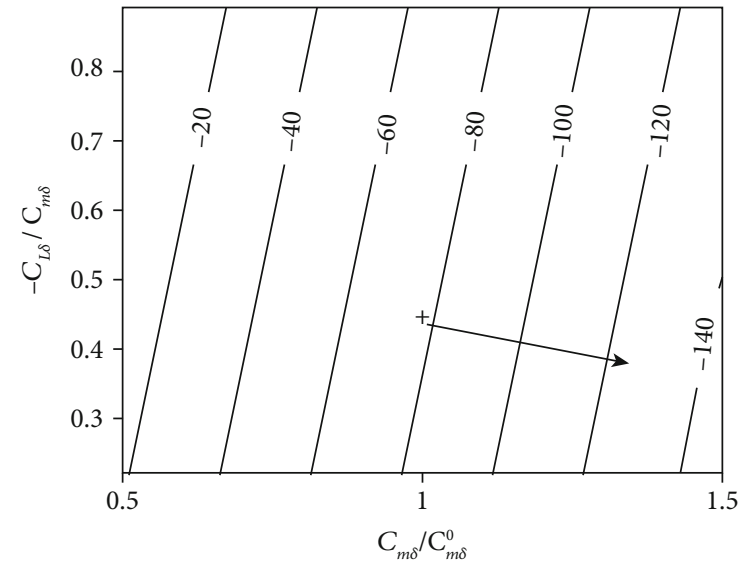

FIGURE 10: Variation in $K_{n}^{\mathrm{min}}$ relative to the control derivatives. +: the baseline model.

distance of centre of gravity from the nose is shown to be $59 \%$ when saturation of deflection of elevators is $\pm 20^{\circ}$, whereas the nominal value is $55 \%$. The primary reason leading to instability issue of HFV dynamics is input saturation. Besides, the control derivatives are also determinant factors affecting the closed-loop stability. The adjustment direction of the aerodynamic characteristics is also indicated to improve control performance. To enlarge the feasible range of static stability region, the control surfaces are expected to be located at the rearward-most position of the vehicle.

\section{Appendix}

The short-period dynamics of Equation (9) can be represented as follows:

$$
\left[\begin{array}{c}
\Delta \dot{\alpha} \\
\Delta \dot{q}
\end{array}\right]=\left[\begin{array}{ll}
a_{11} & 1 \\
a_{21} & 0
\end{array}\right]\left[\begin{array}{l}
\Delta \alpha \\
\Delta q
\end{array}\right]+\left[\begin{array}{l}
b_{1} \\
b_{2}
\end{array}\right] \Delta \delta_{e} .
$$

Expressions of the coefficients in inequality constraints are listed as follows:
(1) Coefficients in Equation (18):

$$
\begin{aligned}
& c_{\sigma, 1}=\left[\begin{array}{c}
-b_{2}-b_{1} \sigma \\
a_{11} b_{2}-a_{21} b_{1}-b_{2} \sigma
\end{array}\right], \\
& c_{\sigma, 2}=\left[\begin{array}{c}
-b_{1} \\
-b_{2}
\end{array}\right], \\
& d_{\sigma, 1}=-a_{21}-a_{11} \sigma+\sigma^{2}, \\
& d_{\sigma, 2}=-a_{11}+2 \sigma .
\end{aligned}
$$

(2) Coefficients in Equation (19):

$$
\begin{aligned}
a_{\zeta} & =\left[\begin{array}{cc}
b_{1}^{2} & b_{1} b_{2} \\
* & b_{2}^{2}
\end{array}\right] \\
c_{\zeta, 1} & =\left[\begin{array}{c}
-2 a_{11} b_{1}-4 b_{2} \zeta^{2} \\
-2 a_{11} b_{2}+4\left(a_{11} b_{2}-a_{21} b_{1}\right) \zeta^{2}
\end{array}\right], \\
c_{\zeta, 2} & =\left[\begin{array}{c}
b_{2} \\
-\left(a_{11} b_{2}-a_{21} b_{1}\right)
\end{array}\right], \\
d_{\zeta, 1} & =-a_{11}^{2}-4 a_{21} \zeta^{2}, \\
d_{\zeta, 2} & =a_{21} .
\end{aligned}
$$

(3) Coefficients in Equation (20):

$$
\begin{aligned}
a_{\omega} & =\left[\begin{array}{cc}
b_{2}^{2}-b_{1}^{2} \omega^{2} & -b_{2}\left(a_{11} b_{2}-a_{21} b_{1}\right)-b_{2} b_{1} \omega^{2} \\
* & \left(a_{11} b_{2}-a_{21} b_{1}\right)^{2}-b_{2}^{2} \omega^{2}
\end{array}\right], \\
c_{\omega, 1} & =\left[\begin{array}{c}
-2 a_{21} b_{2}+2\left(a_{11} b_{1}+b_{2}\right) \omega^{2} \\
2 a_{21}\left(a_{11} b_{2}-a_{21} b_{1}\right)+2 a_{21} b_{1} \omega^{2}
\end{array}\right], \\
c_{\omega, 2} & =\left[\begin{array}{c}
b_{2} \\
-\left(a_{11} b_{2}-a_{21} b_{1}\right)
\end{array}\right], \\
d_{\omega, 1} & =-a_{21}^{2}+\left(2 a_{21}+a_{11}^{2}\right) \omega^{2}-\omega^{4}, \\
d_{\omega, 2} & =a_{21}+\omega^{2} .
\end{aligned}
$$




\section{Data Availability}

The hypersonic model data used to support the findings of this study are included within the article, and the result data are available from the corresponding author upon request.

\section{Conflicts of Interest}

The authors declare that there is no conflict of interest regarding the publication of this paper.

\section{Acknowledgments}

This work was supported by the National Natural Science Foundation of China (grant number 51675264), the Natural Science Foundation of Jiangsu Province for distinguished young scholars (grant number BK20190017), the Jiangsu Planned Projects for Postdoctoral Research Funds (grant number 2019K286), the Six Talent Peaks Project in Jiangsu Province (grant number KTHY-025), the open funds of BISME, and the Fundamental Research Funds for the Central Universities (grant number NS2018052).

\section{References}

[1] B. Chen, Y. Liu, H. Shen, H. Lei, and Y. Lu, "Performance limitations in trajectory tracking control for air-breathing hypersonic vehicles," Chinese Journal of Aeronautics, vol. 32, no. 1, pp. 167-175, 2019.

[2] D. Sziroczak and H. Smith, "A review of design issues specific to hypersonic flight vehicles," Progress in Aerospace Science, vol. 84, pp. 1-28, 2016.

[3] Z. Pu, X. Tan, G. Fan, and J. Yi, "Uncertainty analysis and robust trajectory linearization control of a flexible airbreathing hypersonic vehicle," Acta Astronautica, vol. 101, no. 1, pp. 16-32, 2014.

[4] S. Zhao, X. Li, X. Bu, and D. Zhang, "Prescribed performance tracking control for hypersonic flight vehicles with model uncertainties," International Journal of Aerospace Engineering, vol. 2019, Article ID 3505614, 11 pages, 2019.

[5] X. Bin Zhang and Q. Zong, "Modeling and analysis of airbreathing flexible hypersonic vehicle," Mathematical Problems in Engineering, vol. 29, no. 7, pp. 1205-1210, 2014.

[6] Q. Wu and Y. Guo, "Neural back-stepping control of hypersonic flight vehicle with actuator fault," Journal of Control Science and Engineering, vol. 2018, Article ID 2198423, 5 pages, 2018.

[7] X. Bu, X. Wu, R. Zhang, Z. Ma, and J. Huang, "Tracking differentiator design for the robust backstepping control of a flexible air-breathing hypersonic vehicle," Journal of the Franklin Institute, vol. 352, no. 4, pp. 1739-1765, 2015.

[8] X. Bu, "Guaranteeing prescribed output tracking performance for air-breathing hypersonic vehicles via non-affine backstepping control design," Nonlinear Dynamics, vol. 91, no. 1, pp. 525-538, 2018.

[9] X. Bu, "Envelope-constraint-based tracking control of airbreathing hypersonic vehicles," Aerospace Science and Technology, vol. 95, p. 105429, 2019.

[10] X. Bu, "Guaranteeing prescribed performance for airbreathing hypersonic vehicles via an adaptive non-affine tracking controller," Acta Astronautica, vol. 151, pp. 368-379, 2018.
[11] C. Luo, H. Lei, D. Zhang, and X. Zou, “Adaptive neural control of hypersonic vehicles with actuator constraints," International Journal of Aerospace Engineering, vol. 2018, Article ID 1284753, 15 pages, 2018.

[12] C. Boyi, L. Yanbin, L. Hao, S. Haidong, and L. Yuping, "Stability boundary analysis of hypersonic vehicle with control saturation and bandwidth limitation," Control Theory \& Applications, vol. 33, no. 11, pp. 1508-1518, 2016.

[13] B. Xu and Z. Shi, "An overview on flight dynamics and control approaches for hypersonic vehicles," Science China Information Sciences, vol. 58, no. 7, pp. 1-19, 2015.

[14] M. G. Goman and M. N. Demenkov, "Computation of controllability regions for unstable aircraft dynamics," Journal of Guidance, Control, and Dynamics, vol. 27, no. 4, pp. 647656, 2004.

[15] Z. Yu, Y. Zhang, Z. Liu, Y. Qu, C. Y. Su, and B. Jiang, “Decentralized finite-time adaptive fault-tolerant synchronization tracking control for multiple UAVs with prescribed performance," Journal of the Franklin Institute, 2019.

[16] Z. Yu, Z. Liu, Y. Zhang, Y. Qu, and C. Y. Su, "Distributed finite-time fault-tolerant containment control for multiple unmanned aerial vehicles," IEEE Transactions on Neural Networks and Learning Systems, vol. 31, no. 6, pp. 2077-2091, 2020.

[17] Z. Yu, Y. Qu, and Y. Zhang, "Distributed fault-tolerant cooperative control for multi-UAVs under actuator fault and input saturation," IEEE Transactions on Control Systems Technology, vol. 27, no. 6, pp. 2417-2429, 2019.

[18] L. Saydy, A. L. Tits, and E. H. Abed, "Guardian maps and the generalized stability of parametrized families of matrices and polynomials," Mathematics of Control, Signals and Systems, vol. 3, no. 4, pp. 345-371, 1990.

[19] D. Saussié, L. Saydy, O. Akhrif, and C. Bérard, "Gain scheduling with guardian maps for longitudinal flight control," Journal of Guidance, Control, and Dynamics, vol. 34, no. 4, pp. 1045-1059, 2011.

[20] D. Saussié, L. Saydy, and O. Akhrif, "Longitudinal flight control design with handling quality requirements," Aeronautical Journal, vol. 110, no. 1111, pp. 627-637, 2006.

[21] D. Xiao, M. Liu, Y. Liu, and Y. Lu, "Switching control of a hypersonic vehicle based on guardian maps," Acta Astronautica, vol. 122, pp. 294-306, 2016.

[22] B. Chen, Y. Liu, H. Lei, H. Shen, and Y. Lu, “Ascent trajectory tracking for an air-breathing hypersonic vehicle with guardian maps," International Journal of Advanced Robotic Systems, vol. 14, no. 3, article 172988141770516, 2017.

[23] Standard, Military, Flying qualities of piloted aircraft, US Dept. of Defense MIL-STD-1797A, 1997.

[24] S. Sridharan and A. A. Rodriguez, Multidisciplinary optimization for the design and control of uncertain dynamical systems, PhD diss., Arizona State University, 2014.

[25] S. P. Boyd, L. El Ghaoui, E. Feron, and V. Balakrishnan, Linear matrix inequalities in system and control theory, Society for Industrial and Applied Mathematics, 1994.

[26] J. Brewer, "Kronecker products and matrix calculus in system theory," IEEE Transactions on Circuits and Systems, vol. 25, no. 9, pp. 772-781, 1978.

[27] J. T. Parker, A. Serrani, S. Yurkovich, M. A. Bolender, and D. B. Doman, "Control-oriented modeling of an air-breathing hypersonic vehicle," Journal of Guidance, Control, and Dynamics, vol. 30, no. 3, pp. 856-869, 2007. 
[28] C. Boyi, L. Yanbin, S. Haidong, and L. Yuping, "Surrogate modeling of a 3D scramjet-powered hypersonic vehicle based on screening method IFFD," Proceedings of the Institution of Mechanical Engineers, Part G: Journal of Aerospace Engineering, vol. 231, no. 2, pp. 265-278, 2016.

[29] A. Rodriguez, J. Dickeson, O. Cifdaloz et al., "Modeling and control of scramjet-powered hypersonic vehicles: challenges, trends, and tradeoffs," in AIAA Guidance, Navigation and Control Conference and Exhibit, pp. 1-40, Honolulu, Hawaii, August 2008. 